\title{
NOTE ON 1-DIMENSIONAL INTEGRALLY CLOSED MORI SEMIGROUPS
}

\author{
YOSHIKO KITSUNEZUKA, RYÛKI MATSUDA AND TAKASI SUGATANI
}

\begin{abstract}
We show that a 1-dimensional integrally closed quasi-local Mori semi-group need not be a valuation semigroup, which is a negative answer to a semigroup version of the question of Querré.
\end{abstract}

\section{Introduction}

In [4], Querré states that a 1-dimensional integrally closed quasi-local Mori domain is a discrete valuation domain. As a semigroup version of this statement, the second author posed the question [3, (3.17)]: Is a 1-dimensional integrally closed quasi-local Mori semigroup a discrete valuation semigroup of rank 1? For integral domains, $V$. Barruci [1] showed that a 1-dimensional integrally closed quasi-local Mori domain need not be a discrete valuation domain. Our aim of this short note is to answer the question in the negative.

Notation: Let $S \ni 0$ be a subsemigroup of a torsion-free abelian group, with the binary operation + . Let $q(S)$ denote the quotient group of $S$. A subsemigroup of $q(S)$ containing $S$ is called an oversemigroup of $S$. A mapping $v$ from a torsion-free abelian group $G$ onto a totally ordered additive group $\Gamma$ is called a $\Gamma$-valued valuation on $G$ if $v(x+y)=v(x)+v(y)$ are satisfied for all $x, y \in G$. The subsemigroup $\{x \in G: v(x) \geq 0\}$ of $G$ is called the valuation semigroup of $G$ associated with $v$. A $\mathbb{Z}$-valued valuation is called a discrete valuation of rank 1. A discrete valuation semigroup of rank 1 is the valuation semigroup associated with a discrete valuation of rank 1 . An element $x$ of an extension semigroup $T$ of $S$ is called integral over $S$ if $n x \in S$ for some $n \in \mathbb{N}$. Let $\bar{S}$ be the set of all integral element of $q(S)$ over $S$. Then it is readily seen that $\bar{S}$ is an oversemigroup of $S$. We call $\bar{S}$ the intrgral closure of $S$. If $S=\bar{S}$, then $S$ is called integrally closed. The supremum of the length of the strict chains of proper prime ideals $P_{1} \subset P_{2} \subset \cdots \subset P_{n}$ of $S$ is called the dimension of $S$, which is denoted by $\operatorname{dim}(S)$. For a non-empty subset $A$ of $q(S)$, we put $A^{-1}=\{x \in q(S): x+A \subseteq S\}$, and $\left(A^{-1}\right)^{-1}=A^{v}$. An ideal $I$ of $S$ is called divisorial if $I=I^{v}$. $S$ is called a Mori semigroup if every ascending chain $I_{1} \subset I_{2} \subset \cdots$ of ideals of $S$, which are divisorial, is stationary.

Received November 25, 1998.

1991 Mathematics Subject Classification. 13F30, 13G05, $20 \mathrm{M} 14$.

Key words and phrases. Mori semigroup, valuation semigroup. 
For other unexplained notation, our general reference is [2].

Let $F$ be a torsion-free abelian group, and $H$ a proper subgroup of $F$. We assume that $H$ is integrally closed in $F$. Observe that $\mathbb{Z} \oplus\{0\} \subset \mathbb{Z} \oplus \mathbb{Z}$ is such an extension of semigroups. Let $V$ be a discrete valuation semigroup of $\operatorname{rank} 1$, of the form $F \cup M$, where $M$ is the maximal ideal of $V$. Let $v$ be the valuation associated with $V$. Here is an example satisfying all the properties above: Let $G=F+\mathbb{Z} X$ with a symbol $X$. It is readily seen that $G$ is a torsion-free abelian group. Now define a mapping $v$ from $G$ to $\mathbb{Z}$ by $v(\alpha+n X)=n$ for $\alpha \in F$ and $n \in \mathbb{Z}$. It then follows that $v$ is a discrete valuation on $G$, and $V=F+\mathbb{Z}_{+} X$ is the valuation semigroup associated with $v$, where $\mathbb{Z}_{+}$is the non-negative integers. Further the maximal ideal of $V$ is $M=F+\mathbb{N} X$, and hence $V=F \cup M$. Consider the subsemigroup $S=H \cup M$ of $V$. We have $q(S)=q(V)=G$, and $M$ is the unique maximal ideal of $S$.

Under these preparation, we can prove that $S$ is a 1-dimensional integrally closed quasi-local Mori semigroup that is neither a valuation nor a Noetherian semigroup. We will complete the proof in six steps. In the steps 1 and 2, similar arguments to those of [1] will be used.

Step 1. We show that if $I$ is a non-principal divisorial ideal of $S$, then $I$ is an ideal of $V$. Let $I$ be a non-principal divisorial ideal of $S$. Let $y \in I^{-1}$. It is readily seen that $y+I$ is a proper subset of $S$, since $I$ is not principal. Therefore $y+I$ is contained in the maximal ideal $M$. It then follows that $y+I+V \subseteq M+V=M \subseteq S$. Hence $I^{-1}+I \subseteq S$, or $I+V \subseteq I^{v}$. Since $I$ is divisorial, it follows that $I$ is an ideal of $V$, completing Step 1 .

Step 2. In order to see that $S$ is a Mori semigroup, let $I_{1} \subset I_{2} \subset \cdots$ be an ascending chain of ideals of $S$, which are divisorial. We must show that the chain is stationary. Now Step 1 reduces it to the following two cases: (1) each $I_{n}$ is an ideal of $V$, and (2) each $I_{n}$ is a principal ideal of $S$.

If case (1) happens, then we see that $k_{n}=\min \left\{v(x): x \in I_{n}\right\}$ form a descending sequence $k_{1} \geq k_{2} \geq \cdots \geq 0$. Hence we find $r \in \mathbb{N}$ such that $k_{r}=k_{n}$ for each $n \geq r$. Since each $I_{n}$ is an ideal of $V$, we have that $I_{r}=I_{n}$ for each $n \geq r$.

If case (2) happens, then each $I_{n}$ is of the form $S+a_{n}$ for some $a_{n} \in S$. Then the chain $V+a_{1} \subseteq V+a_{2} \subseteq \cdots$ must be stationary. Let $r \in \mathbb{N}$ be such that $V+a_{r}=V+a_{n}$ for each $n \geq r$. Let $a_{n}=v_{n}+a_{n+1}$ with $v_{n} \in V$. Then we have $v_{n}=a_{n}-a_{n+1}$ is in $S \backslash M$ for each $n \geq r$. Hence $I_{r}=I_{n}$ for each $n \geq r$.

Thus $S$ is a Mori semigroup.

Step 3. We will show that $S$ is not a valuation semigroup. To this end, suppose that $S$ be a valuation semigroup. Choose $\alpha \in F \backslash H$. Then $q(S)=G$ implies that either $\alpha$ or $-\alpha$ is contained in $S$. This in turn shows $\alpha \in H$, a contradiction. Hence $S$ is not a valuation semigroup.

Step 4. In order to see $\operatorname{dim}(S)=1$, let $P$ be a proper prime ideal of $S$. Let $x \in M$. Take $y \in P$. We can find $n \in \mathbb{N}$ such that $v(n x)>v(y)$. It then follows that $n x-y \in M$, and so $n x \in P$. Hence $x \in P$. Thus $P=M$. 
Step 5. To show that $S$ is integrally closed, let $\alpha \in G$ be intrgral over $S$. Since $V$ is a valuation semigroup on $G$, we have $\alpha \in V$. If $\alpha \in F$, then we have $\alpha \in H$, since $H$ is integrally closed in $F$. If $\alpha \notin F$, then $\alpha \in M$. Thus $S$ is integraly closed.

Step 6. It remains to show that $S$ is not Noetherian. To this end we show that $M$ is not finitely generated. For a contradiction we suppose that $M$ is finitely generated. Let $x_{1}, \ldots, x_{n}$ be a minimal generator of $M$ so that $M=\left(S+x_{1}\right) \cup \cdots \cup\left(S+x_{n}\right)$. Note that each $v\left(x_{i}\right)=1$. Now take $\alpha \in F \backslash H$. It is readily seen that $\alpha+x_{1} \in M$, and further $\alpha+x_{1} \notin S+x_{1}$. Hence we have $\alpha+x_{1} \in H+x_{2}$, say. Let $k>1$. We assume that we can arrange the $x_{i}{ }^{\prime} s$ in such a way that $\alpha+x_{i} \notin \bigcup_{j=1}^{i}\left(S+x_{j}\right)$, and $\alpha+x_{i} \in H+x_{i+1}$ for each $1 \leq i \leq k-1$. We want to show that $\alpha+x_{k} \notin \bigcup_{j=1}^{k}\left(S+x_{j}\right)$. If otherwise, then we would find $1 \leq j \leq k$ such that $\alpha+x_{k} \in H+x_{j}$. Then we see that $\alpha+x_{j} \in H+x_{j+1}, \ldots, \alpha+x_{k-1} \in H+x_{k}, \alpha+x_{k} \in H+x_{j}$ yield $(k-j+1) \alpha \in H$. This implies that $\alpha \in H$, since $H$ is integrally closed in $F$. This contradiction completes $\alpha+x_{k} \notin \bigcup_{j=1}^{k}\left(S+x_{j}\right)$. Thus in particular $\alpha+x_{n} \notin M$, the required contradiction.

\section{References}

[1] V. Barucci, On a class of Mori domains, Comm. Alg. 11(1983), 1989-2001.

[2] R. Gilmer, Commutative Semigroup Rings, Univ. Chicago Press, 1984.

[3] R. Matsuda, On some properties between rings and semigroups, Math. J. Ibaraki Uinv. 29(1997), 9-23.

[4] J. Querré, Sur une propriété des anneaux de Krull, Bull. Sci. Math. 95(1971), 341-354.

Department of Mathematics, Toyama University, Gofuku, Toyama 930, Japan.

Department of Mathematical Sciences, Ibaraki University, Mito, Ibaraki 310, Japan.

Department of Mathematics, Toyama University, Gofuku, Toyama 930, Japan. 